\title{
The Spanish CRG instruments at ILL: historical perspective and future developments
}

\author{
Inés Puente Orench, José Alberto Rodríguez Velamazán, Javier Campo \\ Materials Science Institute of Aragón
}

These notes about the activities of the two Spanish Collaborating Research Groups (CRG) at the Institut Laue Langevin (ILL) are written to give a historical view of the group and to present the two new instrumental projects currently in development. After a brief description of the instruments with current Spanish participation (D1B and D15), it will be presented the project of construction of a new detector for D1B as well as the project of building a new Spanish CRG instrument "XtremeD"

\section{Historical review}

D1B turned into a CRG instrument, managed by the Centre National de la Recherche Scientifique (CNRS), in 1997 after being an ILL instrument for more than 20 years. Soon after, in 1998, the Spanish Ministry of Education and Science, through the Spanish Council for Research (CSIC), signed a collaboration agreement with the CNRS for sharing D1B between France (2/3) and Spain (1/3). This guaranteed a preferential access to this instrument for Spanish scientists. In 2008, all these agreements were renewed, with an increase up to $45 \%$ of the Spanish contribution, assuring that D1B will still provide its invaluable services to the Spanish scientific community until 2013. On the other hand, the instrument D15 was a CRG instrument managed by the Commissariat à la Energie Atomique (CEA) since 1996. However, in 2005, the Spanish Ministry of Science signed two agreements, one with the ILL to become CRG manager of D15, and another one with the CEA to share the beam time (60\% for Spain and 40\% for France) of the instrument until 2010 and beyond. The administrative and scientific coordination of the Spanish contribution on both instruments have been done, since the beginnings, through the Materials Science Institute of Aragón (CSIC-University of Zaragoza). Both instruments receive scientists from universities and institutes of CSIC from all around Spain.

One of the main goals of the CRG team is to facilitate the access to ILL instruments to new users. Moreover, the Spanish CRG's favour the access of Spanish scientific groups in which $\mathrm{PhD}$ students are involved. Last but not least, Spanish scientists can benefit of scientific stays at ILL financed by Spain either for preparing or analyzing the data after an experiment.

\section{The powder diffractometer D1B}

D1B is a high flux neutron powder diffractometer placed in a thermal guide of the ILL and equipped with a mobile $3 \mathrm{He} / \mathrm{Xe}$ position sensitive detector (PSD) comprised by an array of 400 cells spanning $80^{\circ}$. A focusing pyrolitic graphite monochromator provides a neutron beam of $2.52 \AA$ with a flux of $6.5 \times 10^{6} \mathrm{n} \mathrm{cm}^{-2} \mathrm{~s}^{-1}$ on the sample position. A second $1.28 \AA$ wavelength is available thanks to a germanium monochromator. 
Experiments at D1B can be carried out under different conditions. There are an orange cryostat and a vanadium furnace especially designed for having low background, which permits to observe small intensities scattered by the sample. Other sample environments of the ILL pool (cryomagnets, pressure cells, dilution cryostats, etc) can be used at the instrument.

D1B was originally designed for studying magnetic structures. However the combination of high flux/low background together with the large detection area makes D1B a suitable instrument for time resolved or in situ neutron diffraction studies. An Eulerian cradle also allows the investigation of texture in polycrystalline samples.

D1B is one of the most productive instruments of the ILL. As an example, during the year 2009, D1B has received 68 scientific groups for carrying out experiments and the amount of publications in 2009 with data taken in D1B is 42. Among them 12 papers are signed by authors with Spanish affiliation. As an example we mention the recent investigation accomplished by Gorria et al. ${ }^{1}$ where "Crystal structure, magnetocaloric effect and magnetovolume anomalies in nanostructured $\operatorname{Pr}_{2} \mathrm{Fe}_{17}$ " are deeply studied. Results reveal that the crystal structure is preserved after milling the sample for 10 hours in a high energy ball mill: However the magnetic anisotropy highly increases due to the drastic reduction in crystalline size.

\section{Single crystal diffraction at D15}

D15 is a single-crystal diffractometer installed in a thermal inclined beam tube. Three wavelengths $\lambda=0.86 \AA, 1.17 \AA$, and $1.54 \AA$ are available from the same $\mathrm{Cu}$ monochromator under a reflection angle $2 \theta=90^{\circ}$ which provides good resolution up to large scattering angles. It can be operated in either the 4-circle geometry or the normal beam geometry and with a wide range of sample environments. Either a standard ${ }^{3} \mathrm{He}$ single detector or an $80 * 80 \mathrm{~mm}^{2}$ micro-strip multi-detector can be used.

The normal-beam mode allows studies with heavy sample environments: orange cryostat, dilution or ${ }^{3} \mathrm{He}$ inserts, pressure cells (up to $100 \mathrm{kbar}$ ), special furnaces, magnetic fields (up to $8 \mathrm{~T}$ ) or combinations of these. The 4-circle configuration allows crystallographic or magnetic studies for temperatures between $2 \mathrm{~K}$ and $700 \mathrm{~K}$.

The principal purposes of D15 are precise crystallographic studies, magnetic structure determinations, studies of phase transitions and H-T/P-T phase diagram determinations, in which it has already proved its competence. The main field of application is the condensed matter area and more specifically magnetism. Recently the scientific range of applications has been broadened with experiments in fields like molecular magnetism, shape memory alloys, Cd-based semiconductors or magnetic multi-layers.

D15 is an up-to-date and well-performing instrument. Unfortunately, it was not operational for the scientific community for almost two years (2005-2006) because of the rebuild of the neighbour instrument IN8, and the need of a new experimental platform satisfying the radiation protection requirements and the new anti-seismic regulations.

${ }^{1}$ Acta Materialia 57, 1724 (2009) 
After these first years of perturbed operation, the access of new groups with different research topics is producing a slow but constant growth of the Spanish scientific production related with D15. As an example of a recent highlight we can mention the study of "Lattice dynamics and external magnetic field effects in Ni-Fe-Ga alloys" by J.I. Pérez-Landazábal, et $\mathrm{al}^{2}$. In this paper, the elastic neutron scattering around Bragg reflections was measured as a function of temperature with $q$-scans along the $[\xi \xi 0]$ direction. These measurements revealed the presence of small satellite peaks at $\xi=0.33$ whose intensity increases on approaching the martensitic transformation temperature. These satellite peaks, together with the inelastic-scattering anomalies confirmed the occurrence of pre-martensitic phenomena in Ni-Fe-Ga alloys.

\section{The new instrumental projects of the Spanish CRG's}

In 2007 the Spanish CRG's team decided to incorporate some technical developments as part of its goals. The present detector of D1B has been running since the 70's and, although it has proved its good quality and performance, there is a big risk of breakdown. Due to that it was decided to accomplish the renewal of the detector in collaboration with the detector group of ILL.

The characteristics of the new detector were defined as follows: the angular range will increase from the present $80^{\circ}$ to $128^{\circ}$, the efficiency will increase up to $80 \%$, and moreover the angular definition will be improved from the actual $0.2^{\circ}$ to $0.1^{\circ}$. All these specifications will produce a gain factor around 5 referred to the old detector. Moreover, the Spanish CRG has undertaken the design and construction of a radial oscillating collimator (ROC).

It is expected to conduct the first commissioning of the detector and ROC during the first cycle of 2011. All the Spanish CRG staff is involved in this project, for which the Spanish Ministry of Science and Innovation (MICINN) has granted $500 \mathrm{k} €$.

On the other hand, the scientific community dedicated to the study of the behaviour of matter under extreme conditions is one of the most actives nowadays. In particular, in Spain there is a strong community dedicated to high-pressure research whose projects in neutron diffraction focus on the crystallography/geosciences area. In addition, the community of magnetism/solid-state sciences, which represents the major part of the Spanish users of ILL, is showing a growing interest for extreme-conditions research, and especially for high-pressure investigations. This interest has led the MICINN to explore the construction of a new Spanish CRG "eXtreme conditions Diffractometer" (XtremeD) for both single crystals and powders.

Apart from the Spanish interest, the ILL and other major groups in Europe dedicated to high pressure investigations have also shown their interest for the XtremeD project by providing representatives to participate as scientific advisors.

Among the different topics in which an extreme conditions diffractometer could contribute, three representative areas can be mentioned:

\footnotetext{
${ }^{2}$ Phys Rev B 80, 144301, (2009)
} 
- Water-related systems: The study of ices and some other water-related systems under extreme pressure conditions implies systematic studies of hydrogen bonding and clustering, host-guest interactions in clathrate hydrates and the role of water in crystals. Understanding such systems has far ranging implications in physics, chemistry, planetary sciences and geochemistry. As an example, current interest in clathrates is motivated by the recognition of their abundance in Earth's subsurface and in icy bodies of the solar system, their potential role in climate changes, and their possible economic importance as a source of fuel.

- Molecular chemistry: In this field, research under extreme conditions tackles a variety of exciting physical phenomena and a number of problems with high social impact, like the improvement of the efficiency in hydrogen storage materials (for example, ammonia borane, $\mathrm{NH}_{3} \mathrm{BH}_{3}$ in the range of $6 \mathrm{GPa}^{3}$ ), the production and characterization of new phases for pharmaceutical applications (polymorphism) or the magnetic and conducting properties of $\mathrm{O}_{2}$, the only elementary molecular magnet. ${ }^{4,5}$

- Magnetism under extreme conditions: A great variety of materials exhibit drastic changes in their magnetic behaviour under high pressures and/or applied magnetic fields: Transition-metal oxides, multiferroics and magnetoelectric materials, frustrated magnetic systems with magnetoelectric properties, magnetic molecular materials, etc. Special attention should be paid to magnetic oxides. The search of novel properties in magnetic oxides is currently a very active area of extraordinary technological importance that covers a great variety of activity domains in which particular magnetic orders and lattice effects are key: from superconductivity to spinelectronics and magnetoelectricity. Neutron diffraction under extreme conditions can make crucial contributions in this field.

The main idea of the instrument XtremeD is to combine a large solid-angle detector with an optional highly focused beam on the sample, thus providing high flux while maintaining low background. The principal features of XtremeD will be:

- Large 2D position-sensitive detector for powders and single crystals

- Optimization for small samples: optional and variable focusing optics

- Radial oscillating collimator and neutron shielding to suppress background

- Sample environment adapted for high pressure (variety of pressure cells, up to $30 \mathrm{GPa}$ for the moment, but with a goal of $50 \mathrm{GPa}$ after the envisaged developments) and high magnetic fields (up to 15 Tesla in continuous mode, with the possibility of higher pulsed fields under study).

- Choice of wavelengths $(0.9 \AA-4 \AA)$ and resolutions. Possibility of having a new supermirror $(\mathrm{m}=3)$ guide in order to expand the wavelength range

The schedule of the project includes a detailed design phase and a construction phase before the commissioning and the exploitation phase foreseen for 2012.

XtremeD will be a CRG managed, like D1B and D15, by the Materials Science Institute of Aragón, most probably with other scientific partners. The proposed CRG framework will provide the necessary flexibility to test new instrumentation developments and to perform difficult and uncertain experiments. The new sample environment developments, combined with advances in neutron science and

\footnotetext{
${ }^{3}$ Y. Lin, W.L. Mao, HK Mao, PNAS, 106, 20, 8113-8116, (2009)

${ }^{4}$ G.Weck, P. Loubeyre, and R. LeToullec, Phys. Rev. Lett. 88, 035504 (2002).

${ }^{5}$ I. N. Goncharenko et al., Phys. Rev. Lett. 93, 055502 (2004)
} 
technology boosted by the construction of such a diffractometer, would open opportunities for new scientific breakthroughs.

The above described characteristics of XtremeD together with its emplacement at the highest neutron flux source, ILL, will make XtremeD an unique instrument in the world 


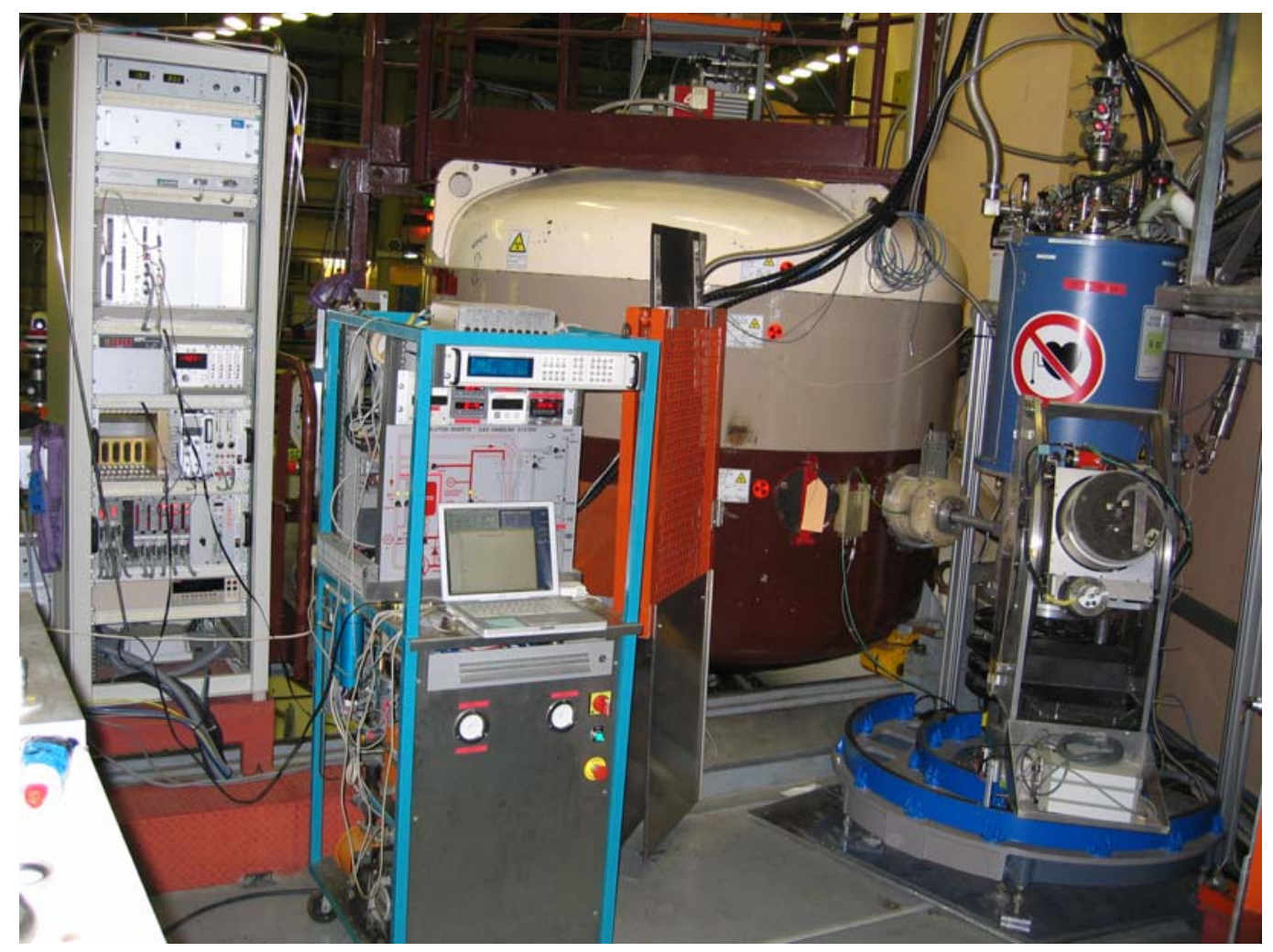

Figure 1. Image of the D15 diffractometer with the dilution insert and cryomagnet set-up:(1) Monochromator casemate; (2) Neutron beam; (3) Dilution insert; (4) 6 Tesla cryomagnet; (5) Detector

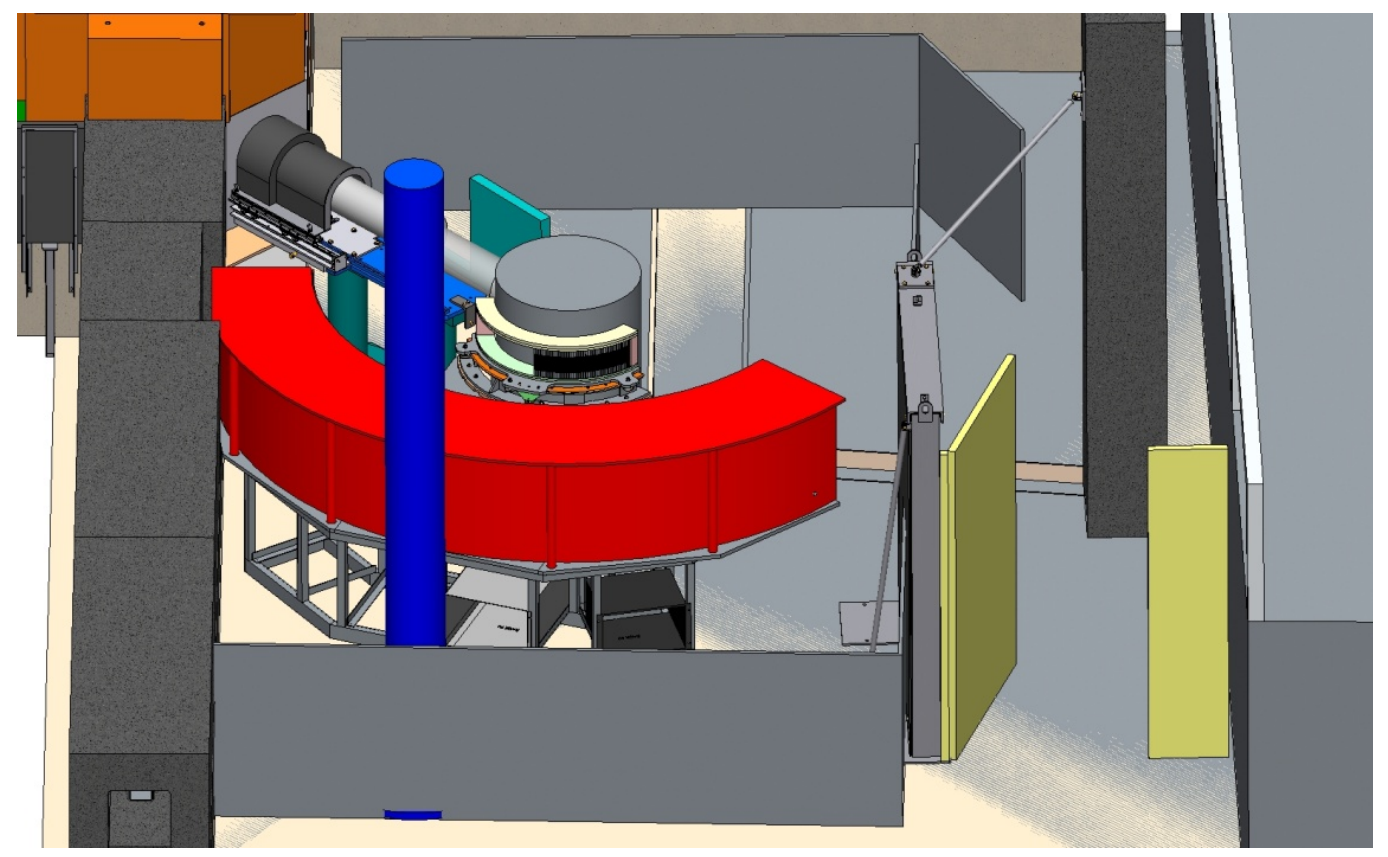

Figure 2. Schematic view of the new detector and ROC for the D1B instrument 